University of Nebraska - Lincoln

DigitalCommons@University of Nebraska - Lincoln

2017

\title{
Assessment with children who need augmentative and alternative communication (AAC): Clinical decisions of AAC specialists.
}

\author{
Shelley Lund \\ University of Wisconsin - Milwaukee, sklund@uwm.edu \\ Quach Wendy \\ San Jose State University, wendy.quach@sjsu.edu \\ Kristy S.E. Weissling \\ University of Nebraska - Lincoln, kristy.weissling@unl.edu \\ Miechelle L. McKelvey \\ University of Nebraska-Kearney, mckelveyml@unk.edu
}

Aimee R. Dietz

University of Cincinnati, aimee.dietz@uc.edu

Follow this and additional works at: https://digitalcommons.unl.edu/specedfacpub

Part of the Analytical, Diagnostic and Therapeutic Techniques and Equipment Commons, Special Education and Teaching Commons, Speech and Hearing Science Commons, and the Speech Pathology and Audiology Commons

Lund, Shelley; Wendy, Quach; Weissling, Kristy S.E.; McKelvey, Miechelle L.; and Dietz, Aimee R., "Assessment with children who need augmentative and alternative communication (AAC): Clinical decisions of AAC specialists." (2017). Special Education and Communication Disorders Faculty Publications. 150.

https://digitalcommons.unl.edu/specedfacpub/150

This Article is brought to you for free and open access by the Department of Special Education and Communication Disorders at DigitalCommons@University of Nebraska - Lincoln. It has been accepted for inclusion in Special Education and Communication Disorders Faculty Publications by an authorized administrator of DigitalCommons@University of Nebraska - Lincoln. 


\title{
Assessment With Children Who Need Augmentative and Alternative Communication (AAC): Clinical Decisions of AAC Specialists
}

\author{
Shelley K. Lund, ${ }^{1}$ Wendy Quach, ${ }^{2}$ Kristy Weissling, ${ }^{3}$ Miechelle McKelvey, ${ }^{4}$ and Aimee Dietz ${ }^{5}$
}

1 University of Wisconsin-Milwaukee

2 San Jose State University

3 University of Nebraska-Lincoln

4 University of Nebraska-Kearney

5 University of Cincinnati

Corresponding author — Shelley K Lund: sklund@uwm.edu

\begin{abstract}
Purpose: The purpose of this study was to explore how speech-language pathologists (SLPs) who are augmentative and alternative communication (AAC) specialists approach the assessment process for 2 case studies, 1 child with cerebral palsy and 1 with autism spectrum disorder. The aim of the study was to answer the following questions: (a) How do clinicians with expertise approach the AAC assessment process for children with developmental disabilities? (b) Can any initial hypothesis be drawn about how SLPs approach the assessment of children with motor versus social interactive deficits? Method: This study used a phenomenological qualitative design. The researchers conducted 2 in-depth, semistructured interviews with 8 SLPs who specialized in AAC and self-identified as primarily working with children.

Results: Four major themes emerged from the data: area of assessment, method of assessment, evaluation preparation, and parent education. Each major theme contained multiple subthemes and categories within those subthemes.

Conclusions: Participants discussed similar areas of assessment for both cases, indicating that some aspects of AAC assessment are universal. However, the specific aspects of what they were assessing and how they went about assessing them differed between the 2 cases. The results of the current study provide an outline of an assessment protocol for children with complex communication needs.
\end{abstract}

Com mprehensive assessment and recommendation of appropriate augmentative and alternative communication (AAC) systems are critical factors in the success of interventions for individuals with complex communication needs (Johnson, Inglebret, Jones, \& Ray, 2006). However, assessing children for AAC systems can be overwhelming for speech-language pathologists (SLPs) who do not perform these assessments on a regular basis (Dietz, Quach, Lund, \& McKelvey, 2012). Various factors contribute to the challenge of completing these assessments: the heterogeneity of individuals who require AAC (Light \& McNaughton, 2012), the large amount of information that must be collected and integrated (Beukelman \& Mirenda, 2013), rapid changes in technology (Abbot \& McBride, 2014; McBride, 2011), and limited research on clinical decision making in AAC assessments (Schlosser \& Raghavendra, 2004).

First, in regard to heterogeneity, the population of individuals with complex communication needs (CCN) who can benefit from AAC is diverse and has changed in recent years. Boyle et al. (2011) indicated that the prevalence of developmental disabilities has increased in the United States. This likely affects the number of individuals that will need AAC services. Light and McNaughton (2012) stated that the number of individuals who require AAC is increasing due to improved survival rates of children born with disabilities and improved life expectancy of those individuals. In addition, they identify that the increased life expectancy of the general population in the United States may lead to a greater chance of an individual requiring AAC in their lifetime. AAC only became an option historically when all hope for speech was lost (Romski \& Sevcik, 2005). Now, we consider AAC options not only to replace spoken language (i.e., as an alternative to speech), but also to supplement (i.e., augment) an individual's speech and facilitate language development (Hustad \& Miles, 2010). This change of philosophy has resulted in an increase in the number of individuals that will require AAC assessments. These increases in numbers coupled with improved awareness and acceptance of AAC contribute to an overall increase in the numbers and types of individuals encountered by SLPs (Light \& McNaughton, 2012). 
SLPs see individuals with a range of disorders requiring AAC services due to $\mathrm{CCN}$. The variety of individuals referred for AAC assessment necessitates the SLP to have knowledge of these disorders, their characteristics, and their progressions (American Speech-Language-Hearing Association, 2002). The underlying etiology can be developmental or acquired; it can also be motor (e.g., cerebral palsy [CP], amyotrophic lateral sclerosis), cognitive/linguistic (e.g., Down syndrome, aphasia), or social interactive (e.g., autism spectrum disorder [ASD]) in nature. Differences in the severity of the disorder also contribute to the complexity of an AAC assessment. For example, no two individuals with $\mathrm{CP}$ will present with the same characteristics.

In addition to heterogeneity, a second challenge for SLPs is the integration of a plethora of data from multiple sources. Collection of data during an AAC assessment may encompass information from parents, teachers, paraprofessionals, peers, ancillary therapists, and school psychologists (Beukelman \& Mirenda, 2013). The method of data collection may vary from clinician to clinician and setting to setting. These procedures are often highly individualized and may be complex and involve many levels of analysis (e.g., multiple environments and partners; McBride, 2011).

A third challenge clinicians face during an AAC evaluation is keeping abreast of the latest technologies. This is particularly true for advances in mobile devices and communication applications, the proliferation of which has increased in the past several years (McNaughton \& Light, 2013). As technology advances, there are a variety of design elements that must be taken into account. Abbot and McBride (2014) described the need to evaluate message, cell, and page layout, which may influence message display and formulation. Consideration of these elements should also include size, number of cells per page, gaps between cells, cell colors, page background colors, and availability/need for keyboard layouts. In addition to design elements, access needs, modes of language representation, types of symbol sets available, and voice elements (e.g., digitized vs. synthesized speech; child vs. adult; male vs. female participants) need to be taken into account during the evaluation process. These technological considerations have to be integrated with the individual's communication needs and abilities. Because technology advances quickly, it is difficult for the clinician to be informed about all the possible software applications, platforms, and dedicated devices available. This need for clinicians to remain current in their understanding of technology adds to the complexity of conducting a comprehensive AAC assessment (Light \& McNaughton, 2012).

The final factor contributing to the challenges of AAC assessment is the paucity of research to support evidence-based practices (Schlosser \& Raghavendra, 2004). Beukelman and Mirenda's (1988) Participation Model was designed as a framework to guide AAC assessment and intervention. The model evaluates communication within the context of an individual's life and includes assessment of five opportunity and to possible access barriers. Whereas the model provides a theoretical framework for AAC assessment, there is little information on how to specifically apply this model in clinical contexts. The decision making that describes how SLPs arrive at their diagnostic conclusions has not been widely or systematically studied. Dietz et al. (2012) discussed the relative discomfort of general practice SLPs while conducting AAC assessments and identified a need for assessment protocols or decision-making guidelines to assist the process. Better understanding of how AAC specialists make clinical decisions is needed to help develop such protocols.

The aim of the current study was to answer the following questions: (a) How do clinicians with expertise approach the AAC assessment process for children with developmental disabilities? (b) Can any initial hypotheses be drawn about how SLPs approach the assessment of children with a motor versus social interactive deficit? To achieve this goal, we explored how eight SLPs who were AAC specialists approached the assessment process for two children, one with a diagnosis of $\mathrm{CP}$ and one with a diagnosis of ASD.

\section{Method}

\section{Research Design}

Due to the complex nature of AAC assessments and the limited research available in this area, a qualitative design was used to explore the research questions. Qualitative methods have been used to examine clinical decision making (Arocha \& Patel, 2007). A phenomenological approach was used because this allowed us to gain a rich understanding of the participants' processes for completing AAC assessments (Cresswell, 2013). We conducted in-depth, semistructured interviews with each participant regarding their approach to AAC assessment for the two cases.

\section{Participants}

Eight certified SLPs, who were native speakers of English, participated in the study. These participants were a subset of the individuals who took part in our previous study on clinical decision making (Dietz et al., 2012). Participants were classified as either AAC clinical specialists $(n=4)$, or AAC research specialists $(n=4)$, according to the Personnel Framework for AAC Assessment (Binger et al., 2012). AAC clinical specialists were defined as persons who provided AAC services during at least $50 \%$ of their clinical workload and had specific skills in AAC assessment. These AAC specialists supported general practice SLPs and others in the AAC assessment process. AAC research specialists were defined as individuals instrumental in preparing future general practice SLPs and AAC clinical specialists. These individuals contributed to the professional knowledge base by carrying out and disseminating research. In addition, AAC research specialists made contributions to AAC clinical practice by creating assessment materials and providing consulting services during AAC assessments. 
To identify potential participants, a purposive sampling procedure (Morse, 2010) was used to ensure that the participants represented each target group. The authors contacted SLPs within their professional networks. The participants filled out a questionnaire about the populations they served, AAC assessment experience, research conducted, and work setting (see online Supplemental Material S1) and were placed in groups on the basis of the results and the definitions previously provided. The participants provided informed consent and are described in this article using pseudonyms to protect their identity.

Demographic information and pseudonyms for the participants are presented in Table 1 . Seven participants were women and one was a man; all were European American. The research and clinical specialists reported similar estimates of workload dedicated to AAC $(M=69.9 \%$ and $71.9 \%$, respectively); however, the clinical specialists conducted considerably more assessments per year $(M=62.5, S D=91.8$, range: 10-200) than the research specialists $(M=15.25, S D=12.0$, range: 5-30). In addition to conducting AAC assessments, research specialists tended to spend the majority of their time teaching and conducting research. They also reported more experience in the field of speech-language pathology $(M=$ 23.5 years, $S D=12.2$, range: $10-37$ ) than clinical specialists $\left(M=11.3\right.$ years, $S D=5.4$, range: $\left.4^{-17}\right)$. The research specialists also had more years of experience in AAC than the clinical specialists $(M=16.5$ years, and $M=11.25$ years, respectively); however, all of the clinical specialists had spent their entire clinical careers focused on AAC.

After collecting 16 samples from the eight participants, no new information emerged from the data, and saturation had been met, indicating the sample size was sufficient (Fusch \& Ness, 2015). The four researchers agreed that there were no significant differences between the two groups, and the data were collapsed into one subject pool.

\section{Materials}

The researchers obtained consent from the parents of two children, one with CP and one with ASD, to use information about their children in the study. The participants received a printed case history form on the basis of a typical form used in university AAC clinics. It included information about the child's medical and developmental history; vision, hearing, cognition, motor, self-help, and communication skills; educational information; adaptive equipment; social information; and therapeutic history. Summaries of each case history are presented in the following sections. An example of the case history form with information from a fictional case similar to one used in the study is presented in online Supplemental Material S2. A brief video clip (child with CP 1:05 minutes; child with ASD 1:20 minutes) of each child from a free-play situation was shown to the participants after they described their initial plan for the assessment. The child with $\mathrm{CP}$ was recorded during a play interaction with a doll using a $3 \times 4$ low-tech communication grid containing Picture Communication Symbols $\left(\mathrm{PCS}^{\mathrm{TM}}\right)$. The child with ASD was recorded during a play interaction with Play-Doh ${ }^{\circledR}$. A DynaVox V ${ }^{\mathrm{TM}}$ with a $5 \times 4$ grid and $\mathrm{PCS}^{\mathrm{TM}}$ symbols was present; however, the child with ASD did not use it during the segment shown to the participants. The video for the child with ASD was digitally recorded in a quiet clinical setting, and the video for the child with $\mathrm{CP}$ was recorded during a home visit. Both recordings were made using a digital video camera mounted on a tripod.

\section{Case History Summaries Ella}

Ella was a 4-year-old girl with athetoid CP. A gastrostomy tube was placed when she was 3 weeks old due to severe oral-motor deficits. She did not walk independently but was able to roll and crawl. Ella's mother reported no vision or hearing

Table 1. Participant demographic information.

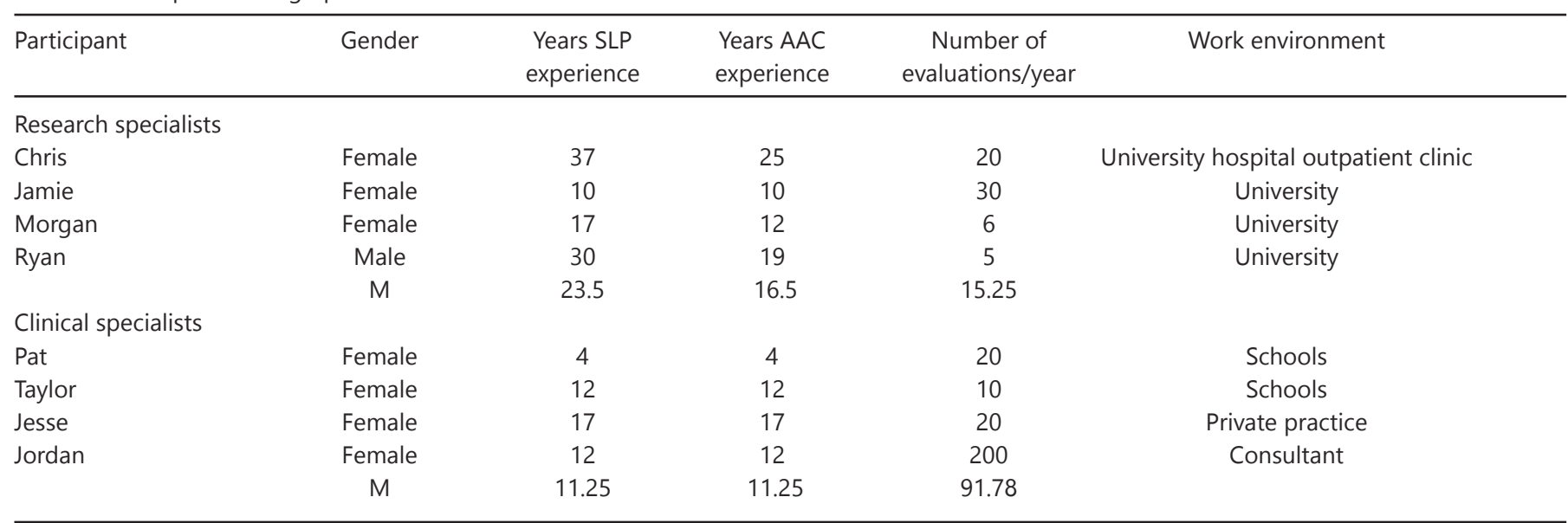

$\mathrm{SLP}=$ speech-language pathologist; $\mathrm{AAC}=$ augmentative and alternative communication . 
problems. In addition, the mother reported that Ella seemed to understand everything said to her. Ella initiated communication readily, was very social, and was eager to communicate. Modes of communication included speech, vocalizations, 8o manual signs (many with modified hand-shapes), and facial expressions. Family and close friends understood Ella's speech, but other people had difficulty understanding her. Ella used communication boards for some play activities and had used a Tech Speak ${ }^{\mathrm{TM}}$ 32-location digitized communication device with $\mathrm{PCS}^{\mathrm{TM}}$ symbols in the past.

\section{Tim}

Tim was a 5-year-old boy diagnosed with ASD, apraxia of speech, and receptive/expressive language disorder. His mother reported that his skills and behavior had regressed at 12 and 18 months of age. Seizure activity was suspected but had not been confirmed. Tim was ambulatory but had difficulty with some fine motor tasks such as fastening buttons and drawing. In reference to his communication, Tim vocalized and used some manual signs but did not use any spoken words. The majority of his communication was achieved by grabbing an adult's hand and leading that adult to what Tim wanted. Earlier, Tim had used the Picture Exchange Communication System $^{\mathrm{TM}}$ (Frost \& Bondy, 2002) and GoTalk ${ }^{\mathrm{TM}}$ 20+ with PCS ${ }^{\mathrm{TM}}$ symbols; however, he no longer used these. His mother reported that Tim had difficulty following directions. Tim was enrolled in an early childhood special education program where he received speech and occupational therapy.

\section{Procedure}

The researchers conducted an in-depth, semistructured interview with each of the participants regarding their approach to AAC assessment for the two cases. Through the interviews, the researchers sought to gain an understanding of the SLPs' rationale regarding the clinical decisions made during two phases of the evaluation process: (a) after reviewing the case history (i.e., the planning portion of the assessment), and (b) after they viewed the client during a short videotaped communicative interaction. The presentation of the two cases was counterbalanced across the participants. The author conducting the interview gave the participant the case history for the first child. After the participant had time to read the history, the author asked, "Can you talk me through your approach to assessing for AAC?" Participants were encouraged to provide additional information using phrases such as "tell me more." After the participant had finished describing their approach, the researcher said, "Now I'm going to show you a brief video clip of " and played the video. After viewing the video, the researcher asked if the participant would do anything differently from what was already discussed. Follow-up questions to seek further information included: (a) "Why or why not?" (b) "What did you see in the video that caused you to change how you would approach the assessment?" (c) "Tell me more about that." The participants completed this process for both cases.

\section{Analysis}

Each interview was audio recorded and orthographically transcribed; a member of the research team then listened to the recordings and corrected any errors in transcription. An inductive coding analysis (Strauss \& Corbin, 1998) was used to allow further understanding of specific domains regarding the participants' experience in conducting AAC evaluations. The transcripts were divided among the researchers for coding. During the initial open-coding process, the researchers conducted a close reading of the text, and codes were assigned to individual thought units (i.e., the smallest amount of information that was meaningful by itself). Second, during selective coding, two researchers grouped similar open codes to develop preliminary themes. Third, all the researchers met as a group to finalize and write definitions for the codes and derive major themes and subthemes. The definitions used for each category are presented in the results section of this article. Last, the first two authors recoded the transcripts independently using these definitions. Afterward, they met to compare their coding; all discrepancies were discussed and recoded to achieve $100 \%$ agreement. Because qualitative coding is an iterative process, the categories and subthemes were reorganized a few times by the authors while summarizing the data until all authors agreed upon the final organization presented here.

\section{Results}

Overall, the research and clinical specialists discussed similar approaches for the two cases, so the results will be presented as a single group. Four major themes emerged from the data: area of assessment (i.e., what was assessed), method of assessment (i.e., how it was assessed), evaluation preparation, and parent education. Table 2 presents a summary of the subthemes and categories, including the number of participants who referred to the categories for each case study. Additional detail and examples of items coded to each category are presented in the following sections.

\section{Area of Assessment (What Was Assessed)}

The theme most frequently discussed by all participants was area of assessment (i.e., what was assessed). This theme related to the specific skills that the SLP proposed to assess for each child. There were three subthemes within this major theme: the individual with complex communication needs (CCN), device features, and partner skills. Large numbers of coding categories were grouped together to form the first two subthemes. All of the categories presented were mentioned by at least two participants.

\section{Individual With CCN}

This subtheme relates to the characteristics and skills of the individual with $\mathrm{CCN}$ that were identified for assessment by the SLP. 
Table 2. Major themes, subthemes, and coding categories represented in the data and the number of participants who discussed the categories for each case.

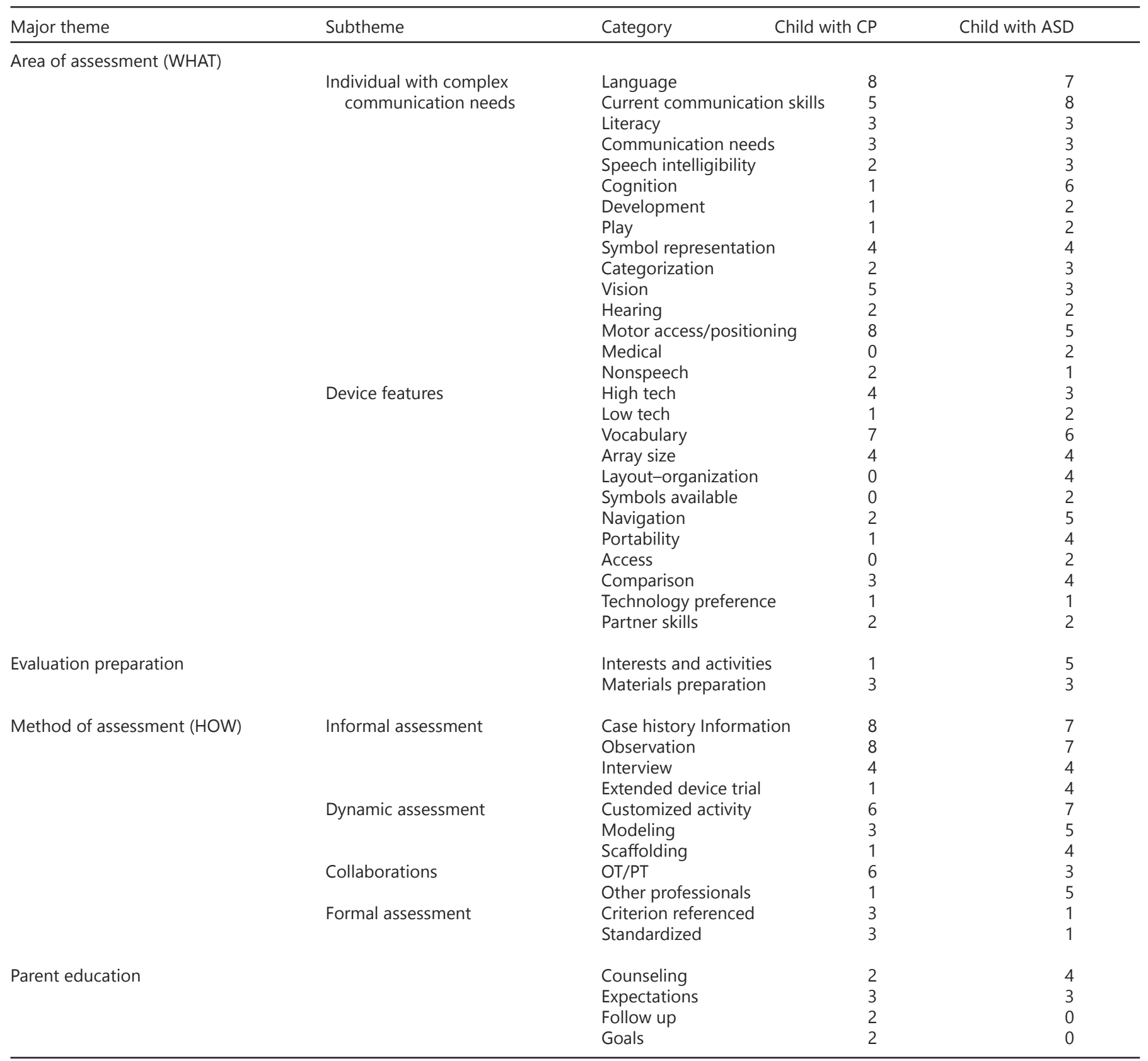

$\mathrm{CP}=$ cerebral palsy; $\mathrm{ASD}=$ autism spectrum disorder; $\mathrm{OT}=$ occupational therapist; $\mathrm{PT}$ = physical therapist.

\section{Language and Communication}

Language. This category included references to seeking information about the individual's language skills with vocabulary/semantics, pragmatics, syntax, or morphology within either expressive or receptive domains. Overall, this category was the most frequently discussed; all participants described aspects of language assessment for the child with $\mathrm{CP}$, and seven addressed issues of language assessment for the child with ASD. Differences emerged regarding the features that participants described as related to each case. Regarding Ella, the child with $\mathrm{CP}$, comments were focused on assessing receptive and expressive language as illustrated in the following quote by Ryan, "I'd want to know more about her receptive language in terms of specifics on vocabulary and syntax and morphological markers, those types of things."

For Tim, the child with ASD, comments were focused on pragmatic aspects of language. The participants described assessing Tim's current use of communicative intents as well as evaluating his ability to learn new intents when provided additional means of communication. The following quote by Chris provides an excellent example of this category "First of all I'm expecting some requesting from him... If I model comments, 
can he expand his pragmatic or social strategies to be able to comment on something."

Current communication skills. This category consisted of information about how the individual currently communicated, including aided and unaided modalities. It also included information about the technology skill level of the individual. This was mentioned for both populations but discussed in depth for Tim, the child with ASD. The participants commented on the variety and range of modalities he used as described in the case history. Jamie summarized it as follows: "He's an intentional communicator, he's grabbing people's hands and leading people to desired objects, etc., and he's got some sign, so we know that he's at least got some emerging expressive symbolic skills, which is great, and he's using speech too- he's got a whole range of communication modes that he's currently using."

Regarding Ella, the child with CP, comments were related to evaluating her ability to use new as well as current modalities. Jesse stated, "I'd like to see how flexible she is. I'd like to see if she naturally starts using vocabulary and symbols in the device, or is she really relying on her signs or her gestures."

Literacy. Information about the individual's ability to understand and use orthography, including reading comprehension and/or spelling, was included in this category. The need to assess literacy skills was discussed equally for both cases. Taylor stated:

"And then also looking at her [Ella's] literacy development and it's nice that she's interested in books, so I'd want to [look] at her phonics really and getting her in an educational program. We could look at that because that could provide a backup later.... I just wouldn't want her to miss out on that literacy side of things."

Ryan also discussed this area, "What's he [Tim] doing for emergent literacy? Does he have letter recognition? You know looking at print recognition type things. If he is a child with ASD maybe he has some hyperlexia. Or maybe he is doing some sight word vocabulary." Given that both children were preschoolers, the focus was on emergent literacy with the goal of moving them to conventional literacy.

Communication needs. This category included information about areas where communication is less than optimal and where additional support is needed. The participants discussed the need to determine the range of communication partners, and level of communicative participation in the children's daily lives. The need to identify and address factors that inhibited communication (i.e., opportunity barriers) was also discussed. The following quotes by Taylor and Ryan, respectively, are typical of the items coded to this category.

\footnotetext{
"When you're only four, you don't communicate that much with strangers. You don't have to go to the shops and [are] not necessarily that communicative with people you don't know. So I would certainly [be] looking at the opportunities to develop her [Ella's] world of people she interacts with." (Taylor)
}

“I don't really see opportunity barriers listed here, but you'd be able to see that if you do the ecological inventories in the different activities. I would start with getting a list of everything he [Tim] does for twenty-four hours a day and picking one or two that are real highneed communication and evaluating those first." (Ryan)

Speech intelligibility. The category of speech intelligibility was considered briefly for both cases. Comments in this category related to information about the perception and clarity of articulation with familiar or unfamiliar partners. Participants discussed the need to determine Ella's intelligibility because the case history reported that she used natural speech with her family. Pat commented, "So the speech assessment. I'm going to look at her intelligibility-it says that she does verbalize and that her parents understand her, so I'll look at her intelligibility at single word level... [with and] without context." Tim was reported to use some vocalizations, so the comments describing assessment for him were focused on determining the range of speech sounds he was using.

\section{Cognition, Development, Play, Categorization, and Symbol Representation}

Cognition. This category included comments seeking information about higher-level thinking skills, memory, problem solving, and ability to take another's perspective. Cognition was discussed exclusively for the child with ASD. Ryan commented, "He [Tim] didn't seem to be attending real well. So I'd like to know, is that typical of him or not?" The SLPs did not discuss any cognitive evaluation for Ella, the child with $\mathrm{CP}$; however, that may have occurred because the case history information indicated that her cognition was within normal limits.

Development. Information about developmental milestones was coded to this category. These comments focused mainly on information presented in the case history form. The following quotes from Taylor illustrate the topics covered in this category, "I'm keen to know her [Ella's] rate of development because ... some of the children I've worked with have sort of plateaued, and I want to know how quickly she was achieving her aims." "I'm impressed he's [Tim] toilet trained...pretty good at four."

Play. Comments in this category reflected observations of or desire to learn more regarding the child's ability and development of play. In reference to Ella, Taylor said, "It would be interesting to see her with other children. I think it'd be really good for her imaginative playing [sic] with other children her age." Pat commented on information in Tim's case history and how it might affect the assessment: "Limited interest in toys makes it interesting too, because that's something you can usually communicate about."

Symbol representation. Assessment of the ability to understand and use graphic representation of meaning was coded to this category. This category was discussed equally for both cases, but surprisingly only half of the participants addressed symbol representation. For Ella, the child with CP, the clinicians felt that a detailed symbol assessment was not needed 
due to her cognitive level as illustrated by Jamie's quote, "I'm not going to be wildly concerned about doing an extensive symbol assessment with her because cognitively she's going to be able to handle what I throw at her." Comments were focused on Ella's higher-level ability to use symbols, as illustrated by the following quote by Pat: "I'd do a quick assessment of whether she can identify multiple meanings for an icon without training. If she knows that apple can mean hungry, eat, and food."

Tim had used some signs and symbols in the past. The participants commented on building upon those skills: Jordan stated, "Mom said pictures, photographs were used, so I think that would be... important as well, to make sure I have that ability to manipulate the types of symbols that are presented."

Categorization. Participants described assessing both children's ability to group concepts with similar features. Jamie stated, "Does she [Ella] understand the categorization 'oh here's where all the people are' and can she go find those taxonomic categories and those kinds of pages?" The focus of this area of assessment was related to determining the way vocabulary was organized in the child's AAC system. Chris commented, "If I give him [Tim] the category symbol for food, can he then go there, and what kind of searching strategies does he use to be able to find that?"

\section{Sensory-Perceptual}

Vision. Comments coded to this category pertained to information about visual acuity and/or perception. This category was discussed equally for both cases but was not a primary focus, because the case histories indicated that both children had vision within normal limits. Pat mentioned the need to monitor Ella's vision due to her CP. "I think most CP kids that I see have some form of vision issue ... The whole coordinating thing, it's hard for them."

Hearing. Coding in this category included information about hearing acuity and/or perception. According to the case histories, both children had normal hearing, so the participants' comments in this category were focused on confirming that their hearing was functional for using a speech generating device (SGD). For example, Morgan stated, "Vision and hearing are not reported to be a problem so I'm probably not going to go too in-depth in that if I don't have any triggers initially."

Motor access/positioning. This was a category frequently addressed and included comments about seeking information about fine motor skills, device access, or positioning. Not surprisingly, all participants discussed motor skills and access for the child with CP. On the basis of the information presented in the case history, the participants thought Ella would most likely use direct selection to access an AAC device. They spoke of using the assessment to fine tune her access needs. Jesse stated, "She's able to direct access but we don't want to do anything that's going to harm her wrists." After observing her positioning in the video clip, the participants often commented on the need to evaluate how Ella's positioning affected her access. Jamie commented, "I'd like to see what she does when she's in a good stable seated position with good trunk support and I bet she can access pretty darn small icons pretty easily."

For Tim, the child with ASD, participants also discussed motor skills but focused mainly on fine motor development, noting some of the information presented in the case history, as demonstrated by Pat: "[The case history] says he has difficulties with buttons, zippers, clasps, things like that, so I'm assuming there's some fine motor coordination issues, which is often seen in autism."

Medical. This category included references to information about concomitant conditions, medications, or diagnoses. Participants commented on information in the case history and wanted to know additional details, as illustrated in the following quote by Taylor, "I would want to be following up on his [Tim's] medical (sic) around seizures.... I'd want to know a little bit more about that and proper diagnosis from a medical doctor."

Nonspeech functions. This category included information about swallowing or other nonspeech functions. As stated in Ella's case history, she was fed by gastrostomy tube. Some participants, such as Taylor, sought more information about her swallowing status: "I would be interested to know [about] her eating and drinking...whether she was having a taste program or whether she was totally unsafe."

\section{Device Features}

Within the theme area of assessment, the second subtheme was device features. This subtheme included characteristics of an AAC system that should be evaluated to determine what is most appropriate for the child. This included consideration or exclusion of specific devices due to these features. There were no significant differences in the frequency of the features discussed in the two cases.

\section{High Tech}

A category within the device features subtheme referenced the need to evaluate or exclude the child's use of high-tech systems. Many participants cited Ella's strong language and cognitive skills as a reason to consider a speech-generating system. Jamie stated, "Assuming that her receptive language and cognition is fairly good, then we're going to go with a high-level, high-end SGD [speech generating device]." Literacy and text generation were also discussed as reasons for considering synthesized versus digitized speech, as illustrated in this quote from Ryan about Tim: "If there's any inkling that there's going to be potential for literacy, and I'm looking at getting a hightech device, that would alter my thoughts on digitized versus synthetic [speech]." However, Taylor expressed the opinion that she would not consider a high-tech system for Ella. “I wouldn't give the little one [Ella] a high-tech device necessarily because I think it could be very distracting and get in the way of some of the play, the learning, the social stuff."

\section{Low Tech}

Items in this category refer to the need for evaluation of the child's use of low-tech systems. Some participants discussed 
low-tech options as a primary strategy to explore, as illustrated by Taylor's comment regarding Ella. "[I'm] more inclined to do like a little communication book with ... color-coded core vocabulary on one side of the book, and fringe vocabulary [on the other]." Other participants discussed investigating low-tech options if the child wasn't able to handle the cognitive load of a high-tech system. After discussing Tim's ability to navigate a dynamic display, Ryan stated: "If he couldn't do that [navigate], then I'd likely back up and try some more simplistic and maybe low tech [options].”

\section{Vocabulary, Array Size, Layout, and Symbols}

Vocabulary. The most frequently discussed category within the device features subtheme was vocabulary. This category pertained to determining the scope of vocabulary needed by the child and any reference to the capacity of a system to store vocabulary or the type of vocabulary included. For example, while discussing Ella's case, Jesse stated, “She seems like she's a prime candidate to have a rich language-based system... make sure that the vocabulary is motivating and meaningful." In reference to Tim, Pat responded, "Once I establish what his [Tim's] receptive language would be, then that can help me decide what type of device...for example, if his receptive language is at least at a 2-year level, and at 2 years we put together two words, he's going to need a core vocab system to at least combine a verb with a noun."

Array size. The comments within this category referred to determining the maximum number of items that should be presented within any single page of the AAC system. Although this feature was discussed for both cases, the specific characteristics of the child guided the participants' decision making and were different. For the child with CP, the participants' were concerned with Ella's motor skills and how that would constrain the selection set, as illustrated by this quote from Jamie: "To get a better feel for the number of symbols, spacing of symbols, that sort of thing, and that's going to be more important for her [Ella] than it is going to be for the other little guy that we looked at [Tim] because of her CP, we need to get a really good idea of what she can handle."

In contrast, for Tim, the child with ASD, the participants were concerned with the cognitive load of the array size. Regarding his previous use of a digitized device, Pat stated: "[It] sounds like he can handle 25 [locations], so I bet he can handle 45 or $50 . "$

Layout-organization. Reference to organization of the vocabulary within an AAC system (i.e., activity, taxonomic, semantic/syntactic, visual scene) was coded to layout- organization. This category is best illustrated by the following quote from Pat, "Then I might consider [system A] that is very categorical...if he's [Tim's] not so strong in categories, then I'm going to have [something] such as a [system B] type program." The participants' comments in layout- organization were often reflective of comments coded in the vocabulary category, demonstrating the link between the content of the vocabulary and the way in which it is organized in the system.
Symbols available. Coding of this category included reference to a device because of its language representation (e.g., graphic symbols, traditional orthography). All of the comments in this category referred to the use of semantic compaction as a representation system. Pat stated, "I'm hoping that he'll [Tim] be able to do some sequencing, which would allow him to do semantic compaction."

Navigation. The items in this category refer to evaluating a person's ability to navigate multiple pages or levels of a communication system as demonstrated in the following quote from Jordan: "See how well she [Ella] can close and navigate out of pages and see in and out of maybe three or four." Jamie stated, "Some of it's going to be how what we see in terms of him [Tim] being able to navigate the device. Can he handle all the different levels you have to go through on [device A]?"

Portability. This category pertained to the size, weight, and maneuverability of an AAC system. Tim was ambulatory, so this was frequently mentioned as a feature to consider for him, as illustrated in the following quote from Pat: "It should be portable. But he is only five. ...it should be light enough that he can carry."

Although Ella wasn't independently mobile, one participant discussed the need for a device to be portable. Taylor commented, "You don't want to give her something big and heavy that's going to get in the way. It would have to be small and lightweight for her."

Access. This category referred to consideration of a device because of its available selection methods. The following quote from Jesse illustrates this category. "If he's [Tim's] just pushing buttons really rapidly, I might increase the hold down time and the selection method a little bit."

Comparison. Any comments referring to comparing the child's performance on two or more AAC systems were coded to this category. Chris discussed comparing device features more generally by indicating a desire to compare screen types: "[We've] gotten to the point where [we've] probably trialed enough of the features to know whether it's important for him to continue with the static display or whether he's going to do well with a dynamic screen system."

The participants discussed the need to compare features between systems. Jesse stated, "I would probably start with [Device $A$ ], and I would have [Device B] and I would probably have one of the smaller systems [available].”

Technology preference. References about child or parent preferences were coded here. Participants discussed integrating preferences into the decision-making process, as the following quote from Jamie exemplifies: "I think it's got to be the confluence of factors so some of it's going to be about him and some of it's about his family. So some families have very strong feelings that they don't like a device or there may be some feature of it that they really want or that sort of thing...so it's not just my decision to make." 


\section{Partner Skills}

Within the theme area of assessment, the third subtheme was partner skills. This subtheme described the evaluation of the child's primary communication partners. The comments here reflected the partners' comfort with technology and interaction skills. Regarding interaction skills, the participants indicated that they would use this part of the assessment to determine appropriate strategies to support the child's social communication and identify potential areas for improvement. The following quote from Taylor about Ella illustrates this subtheme: "I'd really want to look at how her partners interact with her and what strategies that they use that were helpful." Ryan added, "I'd also want to see her interaction with her siblings, and peers as well, and with parents. How do they react to her?"

\section{Evaluation Preparation}

After area of assessment, the second most frequently discussed theme described how the participants would prepare for the evaluation, including information or materials to be gathered prior to seeing the child. The subthemes discussed here include interests and activities, and material preparation.

\section{Interests and Activities}

Interests and activities were discussed most frequently in relation to Tim, the child with ASD. The participants wanted to be prepared with activities that would keep him engaged during the evaluation. Chris stated, "...identifying the things that he really likes to do, that can be used as motivators during the evaluation process...usually if there are primary motivators that the child likes or favorite foods... I request the family to bring those things with them."

\section{Material Preparation}

Comments in this subtheme described the need to obtain equipment for the evaluation, program and customize vocabulary and settings on high-tech systems, or create low-tech materials prior to the evaluation. The participants discussed preparing these materials to ensure that the child had a method with which to communicate during the evaluation, as demonstrated in this quote from Jordan: "It's always important from an access standpoint to make sure that you have a variety of tools, whether it be switches, headmouse, that kind of stuff... even though it says she [Ella] can point, her movement [of] it may be difficult. So maybe some keyguards too. Just to make sure you've got it all."

Jamie discussed preparing communication boards with vocabulary that would be of interest to both children. "I might bring in devices that we mentioned and make sure they're programmed with the different pages that are of interest to him [Tim]" and "...put together context-based boards with an activity that she [Ella] enjoyed."

\section{Method of Assessment (How)}

The third theme addressed the methods that the participants would use to assess the children and obtain the information described in the area of assessment theme. The four subthemes included here, in order of frequency, were informal assessment, dynamic assessment, collaborations, and formal assessment.

\section{Informal Assessment}

This subtheme described evaluation procedures that did not use formal tools. The categories within this subtheme included case history information, observation, interview, and extended device trial. The definitions for each category and illustrative quotes are presented by frequency of occurrence next.

\section{Case History Information}

Items coded to this category included the participants' restatement of information presented in the case history or additional background information desired prior to seeing the child. Chris stated, "The first thing I would do was just what we started with here, looking through the child's case history, identifying any areas of concern." Morgan commented, "There are a number of other professionals listed. I'd want to request the most recent reports from those people: the PT [physical therapist], OT [occupational therapist], and SLP."

\section{Observation}

This category reflected information gathered by watching the child either during the evaluation or in a natural environment. Jamie stated that she would like to have some observational information prior to the assessment: "If she [Ella] came in here for the assessment, I would make sure we had a video of her before we saw her." Chris described how she used observation throughout her assessments: "So I'm always watching visually to see what they're doing as well during part of the assessment."

\section{Interview}

The coding in this category revealed information gathered by asking questions of the child with $\mathrm{CCN}$ or other informants. Jamie gave the following examples: "I can ask Mom questions [about] what she's [Ella] been exposed to literacy wise." "It says he's [Tim's] used the GoTalk ${ }^{\mathrm{TM}}$ in the past, and I'd like to know more about that, what was programmed on that and how he did with it. So that would be one of the questions I [would] ask Mom."

\section{Extended Device Trial}

Codes in this category described the need to engage in a period of diagnostic therapy or extended device loan to evaluate the child's use of an AAC system over time. The participants discussed the need for the children to have extended time to work with different communication strategies before any final recommendations were made. Taylor stated, "I would need to 
trial it...for at least a month." Chris also commented, "[we will determine] whether we need to start working with him [Tim] in therapy for a while to see how he does, give him more exposure first before we start making decisions about whether [he] would need, or he's ready for a communication device."

\section{Dynamic Assessment}

After informal methods, the next most frequently discussed subtheme was dynamic assessment. This included evaluating skills within a functional context and/or manipulating variables to evaluate the effect on the child's performance. Within this subtheme, there were three categories: customized activity, modeling, and scaffolding.

\section{Customized Activity}

Items in this category included participants' descriptions of structured or semistructured activities that they personalized for the children. Most of the activities described were within the context of play activities, such as the following example from Chris: "He's [Tim] interested in stuffed animals, so I might do something with some different stuffed animals that we could have them play or feed them or wrestle or whatever his interest might have been with those."

\section{Modeling}

The second most frequently discussed type of dynamic assessment used modeling, which referred to the participants using AAC strategies during a communicative interaction with the child. The following quote from Jamie exemplified the use of this strategy when introducing AAC technologies: "See if we can get her to do not just two but maybe two, three, or four word messages if we set them up with a Fitzgerald key and do lots of modeling with her and that's going to give us some good information." Jordan added, "A lot of me using the device and modeling and a lot of wait time."

\section{Scaffolding}

Items in the scaffolding category referred to using a graduated or hierarchical cueing strategy to support the child's communication, especially with regard to altering cues to determine which ones provided the child with the best support. For example, Jordan discussed the following regarding Tim's case, “...really looking at that cueing hierarchy. So, at what level do I need to provide him those cues? And I think as you do that more you kind of have a better idea of what's going to be helpful and what's not."

\section{Collaborations}

The next subtheme within the theme of method of assessment refers to conducting the evaluation jointly or in consultation with other professionals.
Occupational and/or Physical Therapists

OTs and/or PTs were the most frequently discussed professions with whom the participants wanted to collaborate. Regarding Ella, the participants were seeking information about seating, positioning, and access. Chris indicated a desire to include an OT and PT: "I would definitely involve an occupational therapist. We may even need to have a PT involved in looking at whether she needs to be supported in a better position."

For Tim, the participants were most concerned with consulting OTs regarding his sensory functioning, as illustrated in this quote from Taylor, "I'd want to get an OT involved. Look at a sensory profile for him-it looks like he's got a lot of sensory issues going on, especially with eating and drinking."

\section{Other Professionals}

Teachers and the school team were included in other professionals that the participants wanted to contact to obtain additional information or to have participate in the evaluation session. Jamie stated, "Discuss very specifically with Mom and the school team the specific ways that he'll [Tim] be able to use the device to communicate more functionally within his classroom setting." Jordan indicated a general desire for additional input, "You know, I think especially in this situation it would have been extremely helpful to get someone else's opinion." However, other participants had more specific individuals from whom they wanted to obtain information. For example, because Tim was receiving applied behavior analysis therapy, some participants were interested in getting information from the psychologist leading the applied behavior analysis treatment team, as illustrated in this quote from Taylor, "I would really want to [talk to] the psychologist."

\section{Formal Assessment}

The final subtheme within the methods of assessment theme was formal assessment. This subtheme included participants' descriptions of using any published assessment tools, whether standardized or criterion referenced.

\section{Standardized}

The participants discussed the use of standardized tests to evaluate receptive language skills, especially for Ella. Tests such as the Peabody Picture Vocabulary Test (Dunn \& Dunn, 2007), the Test of Auditory Comprehension of Language (Carrow-Woolfolk, 2014), and the Clinical Evaluation of Language Fundamentals (Wiig, Semel, \& Secord, 2013) were mentioned. Whereas standardized tests are often used to determine if the child has an impairment, the participants discussed using these measures to determine whether Ella's receptive language skills were within the typical range, as explained by Jamie in the following quote: "We need to do some... formal standardized testing... [to get a] good feeling for what it is she [is] actually comprehending. Is she at age level or isn't she, and if she's not, where is she? And that's going to help us set up our expectations for expressive language goals." 


\section{Criterion Referenced}

This category included statements on the use of tools that were commercially available but not norm-referenced. Criterionreferenced measures were discussed more often than standardized tests. Some of the tools mentioned included social networks (Blackstone \& Hunt Berg, 2012) to assess the child's range of communication partners and modes of communication used, the MacArthur-Bates Communicative Development Inventory (Fenson et al., 2006) to evaluate vocabulary size, or the Test of Aided- Communication Symbol Performance (Bruno, 2010) to evaluate symbol representation.

\section{Parent Education}

The final theme was parent education, which referred to providing information to the parent or family. There were four categories within this theme: counseling, expectations, follow up, and goals.

\section{Counseling}

Comments coded to the counseling category explained the evaluation process and/or device strengths and weaknesses to the family. One participant explained how she would share information about the evaluation process and her decision making with the parents. Chris stated, "I always have families in the room with me. I don't do any evaluation in isolation. I try to talk through what I am doing as I'm doing each part of the assessment...there are no right or wrong answers to this." Jesse said: "I'd probably do a lot of education with the family, saying to them, 'this is a device that I tend to start with for a kiddo like her [Ella],' ...and then really explain to them how the vocabulary in [Device A] is set up and then compare that to the other system."

\section{Expectations}

The participants discussed parents' expectations about the evaluation. For example, in the case history paperwork, Tim's mother indicated that she was interested in procuring a hightech system for her son. Ryan commented on how he would approach this issue with Tim's mother: "She's [Mom] looking at really wanting high tech. I'd want to know what her attitude was about using low-tech as a backup or low tech in other situations."

\section{Follow-Up}

The AAC assessment process often requires multiple sessions over a period of time to complete. The participants stressed the importance of sharing this information with the family, as illustrated in this quote from Jesse: "We'll do all that and you know, we can certainly schedule some follow-up appointments for where that final system recommendation is made."

\section{Goals}

Any references made to inquiring about the individual with $\mathrm{CCN}$ or the family's goals for the assessment and treatment, including recommendations, were included in this subtheme. Jesse commented, "As part of my follow up and talking with Mom, I'm going to highly recommend that she gets her [Ella] into some kind of a preschool program."

\section{Summary of Results}

When describing how they would approach assessment with the two cases provided, the participants described areas of assessment (what they would assess), evaluation preparation, methods of assessment (how they would assess), and parent education. Although there were many similarities between the two cases, the overall emphasis of the assessments for these two children differed. The assessment that the participants described for Ella, the child with $\mathrm{CP}$, was geared to identifying methods to best facilitate her language development and growth. For Tim, the child with ASD, the participants focused their assessment on evaluating his current modes of communication, purposes for communication, and motivation to communicate to determine if he would be able to successfully use AAC strategies.

\section{Interpretation of Results}

The participants in this study described how they would approach assessment for two children, one with $\mathrm{CP}$ and one with ASD, after reading case histories for each child. They discussed similar areas of assessment for both cases, indicating that some aspects of AAC assessment are universal. The areas of assessment that they described included aspects of the Participation Model (Beukelman \& Mirenda, 1988). It is interesting to note that most comments focused on developing a capability profile that described the children's skills and the features to be considered for potential AAC systems. This capability profile is a subset of information to be considered in the access barriers portion of the Participation Model. Not all elements of the Participation Model were discussed by the participants. In particular, information about opportunity barriers emerged less frequently. In addition, little attention was given to assessing the potential to increase natural speech ability or assessing environmental adaptations. These results provide some insights regarding what to assess, method of assessment, evaluation preparation, and parent education. Although the Participation Model focuses on what should be included in an AAC assessment (i.e., opportunity and access barriers) generally, it gives few specifics regarding particular populations.

The results from this study indicate that language is an important area to target in children with $\mathrm{CP}$ and children with ASD. Current communication skills were a focus of assessment in both child cases; however, it was more often discussed in the case involving the child with ASD, Tim. This may be due to children with ASD often having nonconventional modes of communication (e.g., challenging behaviors). 
The domain of cognition emerged as an important area of assessment for Tim, but it was mentioned less frequently in the case of the child with CP, Ella. This result may be due to a focus on the motor impairment in Ella. Assessment of motor skills was mentioned at a more frequent rate in Ella's case than in Tim's case. Next in the area of what to assess, layout organization and portability were addressed more often for Tim. The differences highlighted in the theme of what to assess begin to distinguish the unique focus that clinicians need to consider when assessing a child with $\mathrm{CCN}$ who has a primary motor impairment versus a child with primary social interactive impairment. These results may help to clarify what aspects of the Participation Model (Beukelman \& Mirenda, 1988) may need more emphasis in these populations. Even though the Participation Model offers ideas about what should be assessed, little direction is provided regarding methods of assessment.

During the interviews, four subthemes related to methods of assessment emerged. The participants described informal assessment, dynamic assessment, collaborations, and formal assessment as the means of determining the AAC systems. In the area of informal assessment, the participants relied heavily on case history information and observations. In the area of dynamic assessment, customized activities emerged as a common method for both cases. Scaffolding was mentioned more often with Tim than with Ella. Collaborations were another subtheme emphasized in both populations. However, it appeared that the participants collaborated more frequently with OTs and PTs for Ella and with other professionals (e.g., psychologists, behavior specialists) for Tim. In conclusion, in the subtheme of formal assessment, criteria and standardized assessments were mentioned more often for Ella than for Tim.

The results of this study support the idea that specific approaches to assessment are determined by the characteristics of the client population (i.e., CP and ASD). This indicates that a general outline on how to approach AAC assessment, such as presented in many AAC textbooks, may not be sufficient to help guide SLPs in their clinical practice. Beukelman and Mirenda's (1988) Participation Model coupled with the feature-matching process (Glennen \& DeCoste, 1997) are often considered "best practice" for AAC assessment (Beukelman \& Mirenda, 2013); however, there is little guidance for clinicians with limited expertise on how to clinically apply these models to specific populations of individuals with CCN. The results from this study may form the groundwork for new decision trees and assessment protocols.

\section{Limitations}

The results of this study are based upon a small number of participants who described their approach to AAC assessment with two cases and only reflect the experience of these participants. In addition, the participants in this study were primarily based in the United States. SLPs practicing in different countries may approach AAC assessments differently. The case studies presented were not ethnically diverse, and neither were the participants. Therefore, cultural differences were not captured. All of these factors affect the generalizability of the results.

These data reflect the participants' description of a hypothetical assessment on the basis of the case history information of a single case for each disorder presented. This may not reflect what the participants would actually do with a real client, and it does not reflect the heterogeneity of children with $\mathrm{CP}$ or ASD. Because most of the participants were acquainted with the researcher interviewing them, they may have idealized their performance. In addition, the cases presented did not represent the full range of abilities of children with $\mathrm{CP}$ and ASD, which affects the generalizability of the results because clinicians may encounter children with different characteristics than those represented in this study. These data were collected using interviews, and some participants may have neglected to include all of the procedures they would use in an actual assessment. Future research should incorporate observations of clinicians performing live evaluations to address ecological validity.

\section{Conclusion and Future Directions}

Our previous research highlighted AAC specialists' comprehensive and holistic approach to assessment (Dietz et al., 2012). The results of this study further clarified the methods AAC specialists used to assess children with $\mathrm{CCN}$ for AAC systems, but it also highlighted some of the gaps in current theoretical models of AAC assessment. General practice SLPs have indicated that a protocol to lead them through the AAC assessment process would be beneficial (Dietz et al., 2012). The results of the current study lay the foundation for decision-making guidelines and are an initial step in delineating what should be incorporated into an assessment protocol for children with CCN, specifically, those with CP and ASD.

The results of this study provided information on areas of assessment (what should be assessed), and methods of assessment (how it should be assessed), evaluation preparation, and parent education. However, little information was provided on why clinicians made the decisions they made. It is vital to obtain this information to provide clarity in AAC assessment protocol development. Therefore, future research is needed to understand why AAC specialists make the decisions they do when approaching assessment for different clients, within and across etiologies. This information may be gleaned from a scoping review of the literature or qualitative research methods that study AAC assessments in vivo. 


\section{References}

Abbot, M., \& McBride, D. (2014). Decision making in mobile technology: Points to ponder. SIG 12 Perspectives on Augmentative and Alternative Communication, 23, 104-111.

American Speech-Language-Hearing Association. (2002). Augmentative and alternative communication: Knowledge and skills for service delivery [Knowledge and Skills]. Available from http:// www. asha.org/policy

Arocha, J. F., \& Patel, V. F. (2007). Methods in the study of clinical reasoning. In J. Higgs, M. A. Jones, S. Loftus, \& N. Christensen (Eds.), Clinical reasoning in healthcare professions (3rd ed., pp. 193-203). Sydney, Australia: Elsevier.

Beukelman, D. R., \& Mirenda, P. (1988). Communication options of persons who cannot speak: Assessment and evaluation. In C. A. Coston (Ed.), Proceedings of the National Planners' Conference on Assistive Device Service Delivery (pp. 151-165). Washington, DC: Association for the Advancement of Rehabilitation Technology.

Beukelman, D. R., \& Mirenda, P. (2013). Augmentative and alternative communication: Supporting children and adults with complex communication needs (4th ed.). Baltimore, MD: Brookes.

Binger, C., Ball, L., Dietz, A., Kent-Walsh, J., Lasker, J., Lund, S., . . . Quach, W. (2012). Personnel roles in the AAC assessment process. Augmentative and Alternative Communication, 28, 278-288.

Blackstone, S., \& Hunt Berg, M. (2012). Social networks: An assessment and intervention planning inventory for individuals with complex communication needs and their communication partners. Monterey, CA: Augmentative Communication.

Boyle, C. A., Boulet, S., Schieve, L. A., Cohen, R. A., Blumberg, S. J., Yeargin-Allsopp, M., ... Kogan, M. D. (2011). Trends in the prevalence of developmental disabilities in US children, 1997-2008. Pediatrics, 127 (6), 1024-1042. doi:10.1542/ peds.2010-2989

Bruno, J. (2010). Test of Aided-Communication Symbol Performance. Pittsburgh, PA: Dynavox Mayer Johnson.

Carrow-Woolfolk, E. (2014). Test of Auditory Comprehension of Language (4th ed.). Austin, TX: Pro-Ed.

Cresswell, J. W. (2013). Qualitative inquiry and research design: Choosing among five approaches (3rd ed.). London, UK: Sage.

Dietz, A., Quach, W., Lund, S. K., \& McKelvey, M. (2012). AAC assessment and clinical-decision making: The impact of experience. Augmentative E Alternative Communication, 28, 148-159. doi:10.3 109/07434618.2012.704521

Dunn, L., \& Dunn, D. (2007). Peabody Picture Vocabulary Test (4th ed.) San Antonio, TX: Pearson Education.

Fenson, L., Marchman, V., Thal, D., Dale, P., Reznick, S., \& Bates, E. (2006). The MacArthur Bates Communicative Development Inventories (2nd ed.). Baltimore, MD: Brookes.
Frost, L., \& Bondy, A. (2002). The Picture Exchange Communication System training manual (2nd ed.). Newark, DE: Pyramid Educational Products.

Fusch, P. I., \& Ness, L. R. (2015). Are we there yet? Data saturation in qualitative research. The Qualitative Report, 20, 1408- 1416. Retrieved from http://www.nova.edu/ssss/QR/QR2o/9/ fusch1.pdf

Glennen, S., \& DeCoste, D. (1997). The handbook of augmentative and alternative communication. San Diego, CA: Singular.

Hustad, K. C., \& Miles, L. K. (2010). Alignment between augmentative and alternative communication needs and school-based speechlanguage services provided to young children with cerebral palsy. Early Childhood Services, 4, 129-140.

Johnson, J. M., Inglebret, E., Jones, C., \& Ray, J. (2006). Perspectives of speech language pathologists regarding success versus abandonment of AAC. Augmentative and Alternative Communication, 22, 85-99.

Light, J., \& McNaughton, D. (2012). The changing face of augmentative and alternative communication: Past, present, and future challenges. Augmentative and Alternative Communication, 28, 197-204.

McBride, D. (2011). AAC evaluations and new mobile technologies: Asking and answering the right questions. SIG 12 Perspectives on Augmentative and Alternative Communication, 20, 9-16.

McNaughton, D., \& Light, J. (2013). The iPad and mobile technology revolution: Benefits and challenges for individuals who require augmentative and alternative communication. Augmentative and Alternative Communication, 29, 107-116.

Morse, J. M. (2010). Sampling in grounded theory. In A. Bryant \& K. Charmaz (Eds.), The SAGE handbook of grounded theory (pp. 229-244). London, UK: Sage.

Romski, M., \& Sevcik, R. A. (2005). Augmentative communication and early intervention: Myths and realities. Infants $\mathcal{E}$ Young Children, 18(3), 174-185.

Schlosser, R. W., \& Raghavendra, P. (2004). Evidence-based practice in augmentative and alternative communication. Augmentative and Alternative Communication, 20(1), 1-21.

Strauss, A., \& Corbin, J. (1998). Basics of qualitative research: Techniques and procedures for developing grounded theory (2nd ed.). Thousand Oaks, CA: Sage.

Wiig, E., Semel, E., \& Secord, W. (2013). Clinical Evaluation of Language Fundamentals (5th ed.). San Antonio, TX: Pearson Education. 